\title{
PROJECTING THE RISK OF FUTURE CLIMATE SHIFTS
}

\author{
DAVID B. ENFIELD ${ }^{\mathrm{a}, *}$ and LUIS CID-SERRANO ${ }^{\mathrm{b}}$ \\ ${ }^{a}$ NOAA Atlantic Oceanographic and Meteorological Laboratory, Miami, FL 33149, USA \\ b Statistics Department, Universidad de Concepción, Concepción, Chile \\ Received 9 August 2005 \\ Revised 8 November 2005 \\ Accepted 9 November 2005
}

\begin{abstract}
Recent research has shown that decadal-to-multidecadal (D2M) climate variability is associated with environmental changes that have important consequences for human activities, such as public health, water availability, frequency of hurricanes, and so forth. As scientists, how do we convert these relationships into decision support products useful to water managers, insurance actuaries, and others, whose principal interest lies in knowing when future climate regime shifts will likely occur that affect long-horizon decisions? Unfortunately, numerical models are far from being able to make deterministic predictions for future D2M climate shifts. However, the recent development of paleoclimate reconstructions of the Atlantic Multidecadal Oscillation (AMO) (Gray et al., 2004) and Pacific Decadal Oscillation (PDO); (MacDonald and Case, 2005) give us a viable alternative: to estimate probability distribution functions from long climate index series that allow us to calculate the probability of future D2M regime shifts. In this paper, we show how probabilistic projections can be developed for a specific climate mode - the AMO as represented by the Gray et al. (2004) tree-ring reconstruction. The methods are robust and can be applied to any D2M climate mode for which a sufficiently long index series exists, as well as to the growing body of paleo-proxy reconstructions that have become available. The target index need not be a paleo-proxy calibrated against a climate index; it may profitably be calibrated against a specific resource of interest, such as stream flow or lake levels. Copyright (c) 2006 Royal Meteorological Society.
\end{abstract}

KEY WORDS: Atlantic multidecadal oscillation; climate impacts; risk projection

\section{INTRODUCTION}

The last 15 years have seen much research on decadal-to-multidecadal (D2M) climate modes and their global and regional impacts. The history of D2M has gone through several overlapping phases. The earliest, and still ongoing, is the model- and observation-based inquiry into the nature and causes of D2M climate variability with papers on the El Niño-Southern Oscillation (ENSO)-like Pacific Decadal Oscillation (PDO; Gu and Philander, 1997; Mantua et al., 1997), a possibly related Interdecadal Pacific Oscillation (IPO; Folland et al., 1999; Power et al., 1999), the Arctic Oscillation (AO; Thompson and Wallace, 1998), the North Atlantic Oscillation (NAO; Hurrell, 1995; Marshall et al., 2001) and the Atlantic Multidecadal Oscillation (AMO; Schlesinger and Ramankutty, 1994; Andronova and Schlesinger, 2000; Delworth and Mann 2000; Latif et al., 2004). In the specific case of the AMO applied in this study, the evidence from coupled models suggests that the Atlantic overturning circulation is implicated in the AMO mechanism (Delworth and Mann, 2000; Knight et al., 2005).

Another phase of research comprised papers demonstrating that at least some of these D2M modes suggest compelling manifestations in climatic and ecological impacts. This is especially notable for precipitation and drought frequency, which appear to be sensitive to small but persistent changes in the prevalent atmospheric circulation patterns over the continental regions adjacent to the oceans that mediate the oscillations. Impacts

\footnotetext{
* Correspondence to: David B. Enfield, NOAA Atlantic Oceanographic and Meteorological Laboratory, Physical Oceanography Division, Miami, FL 33149, USA; e-mail: David.Enfield@noaa.gov
} 
have been identified for the PDO (Mantua et al., 1997), the NAO (Marshall et al., 2001) and the AMO (Enfield et al., 2001), and most recently for the interaction between the PDO and AMO modes (McCabe et al., 2004). Moreover, both the PDO and the AMO are shown to modulate (render nonstationary) the rainfall signatures of ENSO in the United States (Gershunov and Barnett, 1998; McCabe and Dettinger, 2000; Enfield et al., 2001). Enfield et al. (2001) estimate that the inflow to Lake Okeechobee (south Florida's water supply reservoir) undergoes a $40 \%$ peak-to-peak variation associated with the AMO. Another recently identified area of strong impacts is the AMO modulation of major Atlantic hurricane activity (Goldenberg et al., 2001), while Mantua et al. (1997) describe the basin-wide impact of the PDO on Pacific fisheries such as salmon. From the response of an atmospheric global circulation model (AGCM) to observed Atlantic sea surface temperature (SST), Sutton and Hodson (2005) have confirmed the AMO climate impacts suggested by the limited data available (Enfield et al., 2001; McCabe et al., 2004).

One of the greatest difficulties for climate researchers is the fact that even the longest instrumental time series are frequently too short to extract conclusions about D2M variability with satisfactory statistical confidence. Fortunately, there is a growing body of paleoclimatic work - especially that based on tree rings - which has extended our temporal reach for centuries or more into the past, and which faithfully reconstructs either the instrumental climate mode indices or specific responses to the modes, such as streamflow (Woodhouse et al., 2006).

Of concern for climate applications is the fact that - unlike ENSO - numerical models have so far proven incapable of predicting future phase shifts of D2M climate modes in a deterministic manner. The alternatives to such predictions are probability-based projections. However, probabilistic projections are hampered because the instrumentally-based time series are limited to the last 130-150 years at most, which yield too few realizations of $\mathrm{D} 2 \mathrm{M}$ cycles for conventional statistical approaches to deal with. In this study, we resolve the problems associated with small sample sizes for a specific climate mode, the AMO, in two ways: (1) by applying Monte Carlo-style resampling techniques to the index data and (2) by application to the longer, 424-year paleoclimate reconstruction based on tree rings (Gray et al., 2004). By then adjusting a probability model to the distribution of resampled AMO phase intervals, we extract a practical method for determining the probability of a future departure from the current AMO climate regime. In lieu of nonexistent deterministic predictions, this method provides an essential element for the development of decision support tools for managers and stakeholders in sectors affected by D2M climate modes, such as agriculture, water, energy, health and disaster risk. For probabilistic projection to be useful, we do not require an understanding of the physical mechanisms governing the climate mode or its impacts, only a reasonable certainty that the impacts of interest derive from the climate mode in question. Even the latter is irrelevant if the methods are applied directly to an index of the impact itself, such as a dendrochronological reconstruction of stream flow feeding a water management system.

\section{DATA AND METHODS}

We use two unsmoothed data sets to index the AMO: an updated (1856-2001) annualized version of the instrumentally-based AMO index suggested by Enfield et al. (2001), and the 424-year annualized index of the AMO reconstructed from tree rings in North America and Europe (Gray et al., 2004). The former index is the demeaned and detrended SST averaged for the North Atlantic Ocean from the equator to $70^{\circ} \mathrm{N}$. The Gray et al. index is the longer tree-ring series calibrated against the instrumental index.

To discourage unwanted short-interval occurrences, the time series are smoothed with a Butterworth filter of order 8 and a half-amplitude response cutoff at 15 years. The filter is passed forward and backward to remove unwanted phase effects. The Butterworth filter is an infinite impulse response filter commonly used in signal analysis applications, which has monotonic rolloff and very reduced ripple effects in the pass band and stop band, making it more desirable than the running boxcar filters used in many climate applications. Order 8 achieves less than $3 \mathrm{db}$ of ripple at periods greater than 20 years, and at least $40 \mathrm{db}$ of attenuation at periods below 10 years. 
To increase the sample size, the method of Ebisuzaki (1997) is used to randomly resample the series multiple times, and the durations of positive and negative phases are estimated by the intervals between successive zero crossings of the resampled series. The empirical distributions of sample intervals are then fitted by a gamma probability density function (pdf) and a Kolmogorov-Smirnov (KS) test is used to determine the goodness of fit. The resampling and fitting procedure is repeated $N=50$ times to obtain stable means of the gamma distribution parameters for scale and shape. However, choice of $N$ is arbitrary and was chosen here by observing the convergence of parameter values as $N$ increases. The gamma fit for the longer, Gray et al. series is used to construct the probability of future AMO shifts conditional on the time elapsed since the last shift. Finally, the stationarity of the Gray et al. series is tested by repeating the procedure for three shorter, 141-year segments of the data, then comparing the distribution parameters obtained. The spread of resulting gamma parameters is used to estimate the uncertainty of the probability projections.

\section{RESAMPLING PROCEDURE}

Figure 1 (a) shows the smoothed AMO reconstruction of Gray et al. (2004), annotated with the intervals between zero crossings, plus similar plots for three randomly resampled versions of the data. The Ebisuzaki (1997) 'random phase' method of resampling consists of transforming the original time series into the frequency domain, randomizing the Fourier phases, and reverse transforming to the time domain. Unlike most randomizations in the time domain, this method preserves the original power spectrum but still produces resampled series whose temporal correlations with each other and the original series are expected to be zero on average. When the resampling is repeated many times, the resulting autospectra form a two-standard deviation envelope that brackets the spectrum of the original data to a good approximation (Figure 2). The assumption

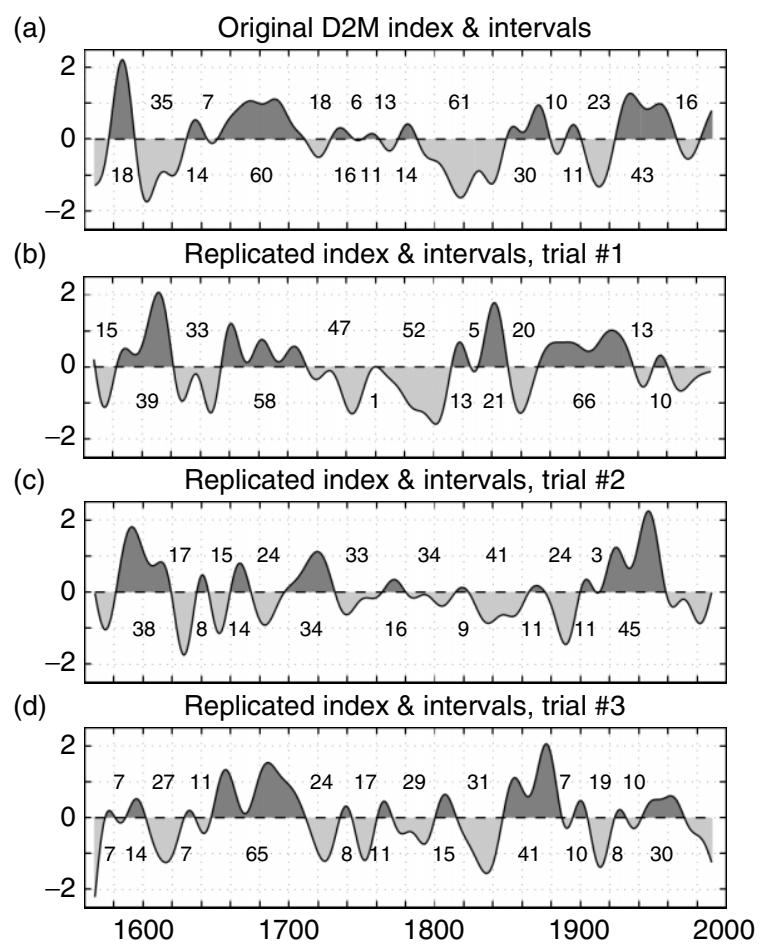

Figure 1. (a): Smoothed annual tree-ring reconstruction of the Atlantic Multidecadal Oscillation (AMO) index by Gray et al. (2004). (b, c, d): Smoothed resampled versions of the Gray et al. Index using randomization in the frequency domain (Ebisuzaki, 1997). Numeric annotations are the intervals (years) between zero crossings 


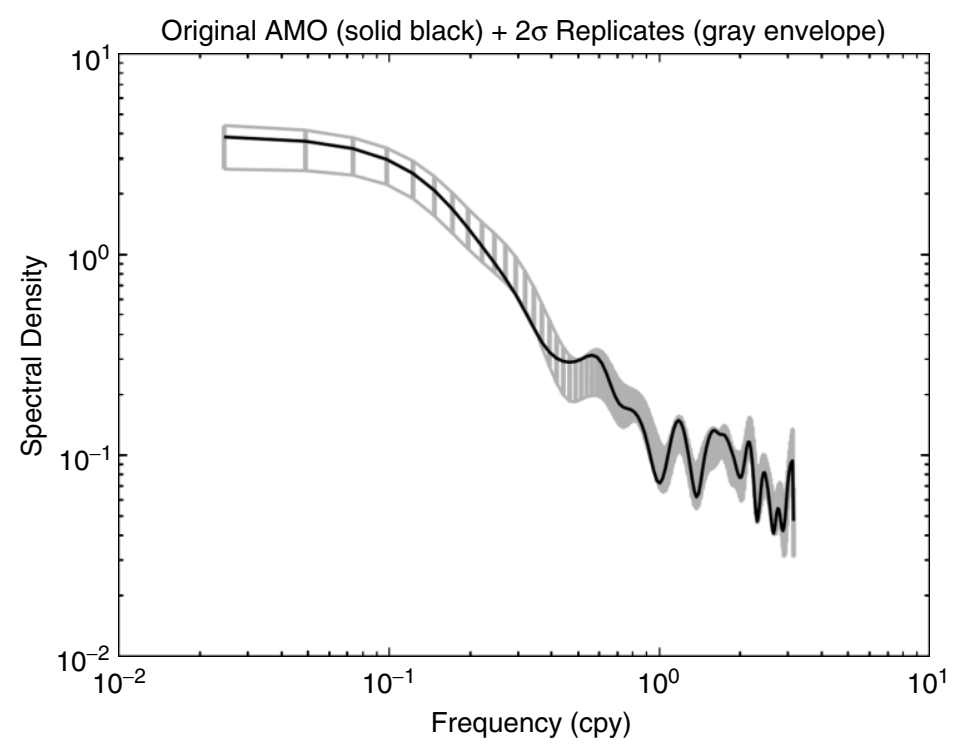

Figure 2. Black curve: Autospectrum of the unsmoothed Gray et al. (2004) index. Light-shaded envelope: mean \pm 2 standard deviations of the spectral energy at each frequency for 50 resampled versions of the unsmoothed Gray et al. index

implicit in this resampling is that the original series is extracted from a larger population (longer duration) with time-invariant statistics (stationary). The question of stationarity will be examined in a later section.

As seen in Figure 1 (a), the original time series resolves 18 AMO phase intervals between the midsixteenth century and the late twentieth century. To obtain a large enough sample for fitting purposes (about 100), we resample five times. A primary difference between increasing the sample size spectrally versus a bootstrap resampling of the original 18 intervals (Efron, 1979) is that the latter method produces a sample whose members have numerical values extracted from and limited to the original 18, whereas the spectral randomization extracts entirely new values from the same power spectrum signature. With the bootstrap, it is critical that the original sample be large enough to be representative of the larger population from which it arises, while with the Fourier approach, it is sufficient that the spectrum be representative of the D2M process.

\section{DISTRIBUTION FITTING}

The histogram of Figure 3 (a) illustrates a typical empirical distribution of AMO regime intervals produced by extracting five new time series from the original Gray et al. (2004) spectrum. The distribution is fit by the smooth curve, which corresponds to a gamma pdf whose shape (A) and scale (B) parameters are adjusted to the data by maximum likelihood estimation (MLE). As in the example shown, a KS goodness-of-fit test is applied to the cumulative distribution (cdf, lower panel) and usually shows the fit to be acceptable at the $95 \%$ level of significance. Each new fivefold resampling results in varied but similar parameter estimates. To obtain a stable estimate of the gamma distribution for the 424-year period, we average the parameter estimates from 50 resamplings, obtaining $A=1.93$ and $B=10.3$. These values are later used to project the risk of future regime shifts.

\section{STATIONARITY TEST}

We now examine the hypothesis implicit in the above calculations, namely, that the 424-year reconstruction arises from a stationary process. We define stationarity to mean that the distribution parameters $A$ and $B$ are acceptably invariant in time - in this case, that the confidence intervals about the parameter means overlap between temporally adjacent samples. Since we have no additional data 

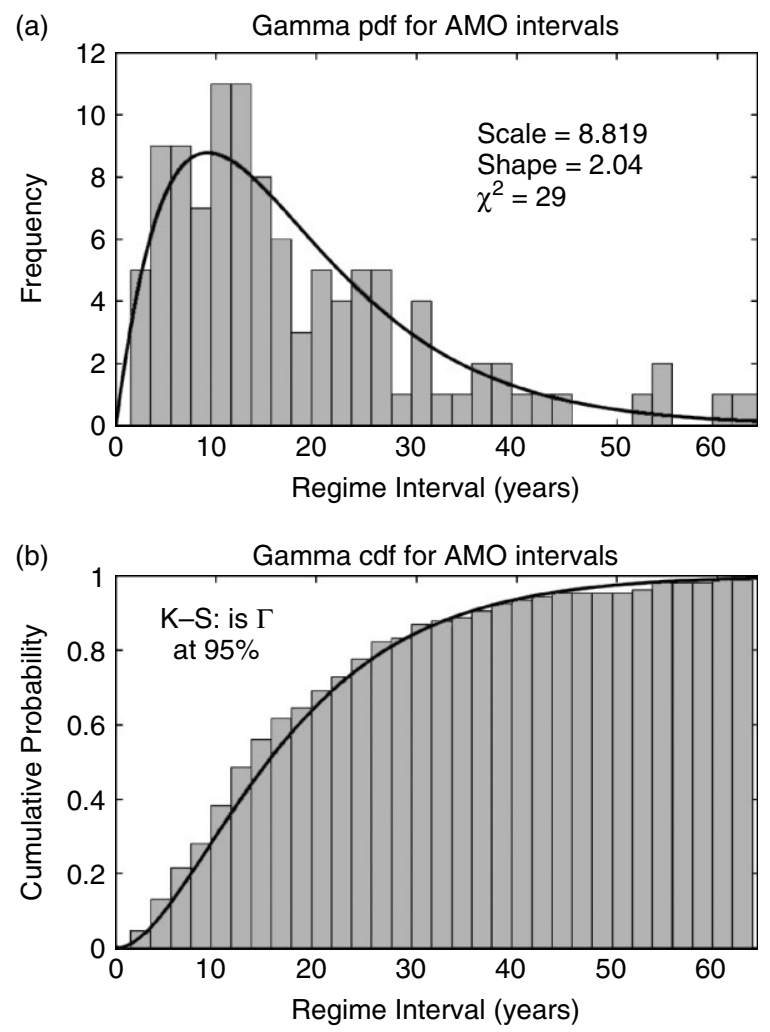

Figure 3. (a): Histogram (vertical bars) of zero crossing intervals from a set of five resampled and smoothed versions of the Gray et al. (2004) index and the maximum likelihood (MLE) gamma probability distribution (solid curve) fit to the histogram. (b): Cumulative empirical distribution (vertical bars) and gamma cumulative distribution function (solid curve), indicating that the Kolmogorov-Smirnov goodness-of-fit criterion is satisfied at the $95 \%$ significance level

before or after the Gray et al. reconstruction to test this on, we instead divide the Gray et al. series into three, nonoverlapping, 141-year segments and recalculate the 50-member ensembles for those segments and compare their parameter spreads. Because we also have the instrumental series with a similar length, which coincides with the third Gray et al. segment, we also repeat the procedure for that series. This produces four ensembles of parameter estimates, that is, 50 values of $A$ and $B$ for each of the four segments tested. These ensembles are summarized by the box-and-whiskers plots of Figure 4.

Not only the interquartile ranges but also the whiskers (all parameter estimates except for outliers) are nonoverlapping; hence, we can safely reject the stationarity hypothesis because the whiskers embrace more than $95 \%$ of the values. The 424-year parameter means (long horizontal lines) are bracketed by the segment estimates, especially those after A.D. 1700. The instrumental AMO index also falls within the range of the Gray et al. segments. Hence, we conclude that the AMO process is nonstationary, that the instrumental and reconstruction segments are statistically compatible, and that the 424-year mean distribution provides a reasonable basis for making projections. The fact that the process is nonstationary does not invalidate the estimation procedure, but it means that the distribution parameters are more uncertain than implied by the 50-member spread for the longer 424-year estimation. We shall return to this later.

The segment-to-segment variation of the interval means is not obvious from Figure 4 because the population mean for each gamma fit is the product of the distribution parameters, $\mu=A^{*} B$. For the three Gray et al. data segments, the ensemble estimates of the distribution means are 17.3, 16.4 and 18.7 years, respectively, with $95 \%$ confidence intervals ranging from 0.22 to 0.28 years. Hence, the regime intervals for the middle 

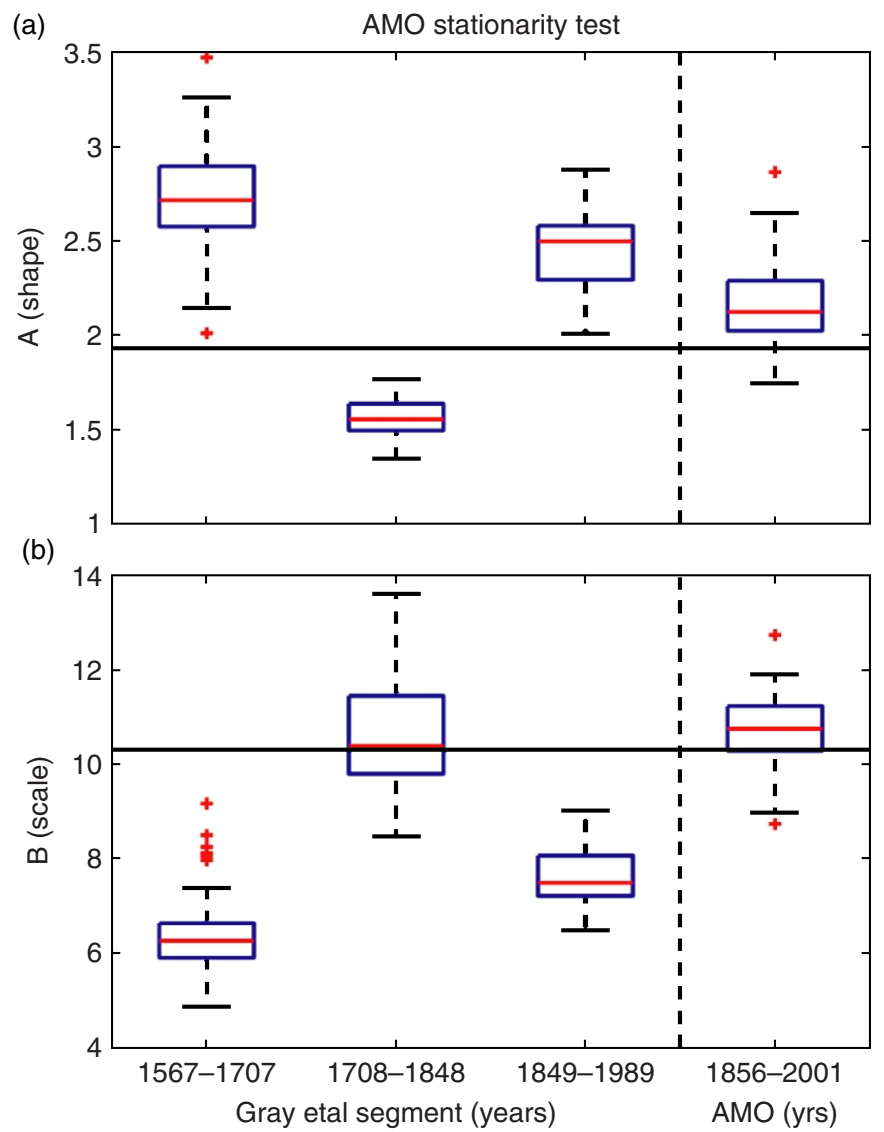

Figure 4. Box-and-whiskers plots for four 50-member sets of shape (a) and scale (b) parameter estimates. Those for the three 141-year segments of the Gray et al. reconstruction are shown to the left of the vertical dashed line. Those for the instrumental AMO index are show to the right of the line. Each box has horizontal lines at the lower quartile, median, and upper quartile values. The whiskers are vertical lines extending from the boxes to show the extent of the rest of the data. Outliers are + symbols beyond the ends of the whiskers. The long horizontal line in each panel marks the mean value of the 50-member ensemble for the entire 424-year reconstruction. This figure is available in colour online at www.interscience.wiley.com/ijoc

segment (1708-1848) are significantly shorter than before or after. This is qualitatively consistent with the character of the tree-ring reconstruction, which shows the dominance of longer intervals near the start and end of the series (S. Gray, personal comm.).

\section{PROBABILITY PROJECTIONS}

If we let $P(\rho)$ represent the probability of a realization $\rho$ within the population space of the stochastic regime intervals $(T)$, we can then construct useful probability projections for future realizations. For example, the conditional probability that a future regime shift will occur within a horizon of $t_{2}$ years, given that $t_{1}$ years have elapsed since the last, opposite regime shift, may be expressed as

$$
\begin{aligned}
P\left(T>t_{1} \cap T \leq t_{1}+t_{2} \mid T>t_{1}\right) & =P\left(T>t_{1} \cap T \leq t_{1}+t_{2}\right) / P\left(T>t_{1}\right) \\
& =P\left(t_{1}<T \leq t_{1}+t_{2}\right) / P\left(T>t_{1}\right) \\
& =\left(\Gamma\left[t_{1}+t_{2}\right]-\Gamma\left[t_{1}\right]\right) /\left(1-\Gamma\left[t_{1}\right]\right)
\end{aligned}
$$


where $t=t_{1}+t_{2}$ is the current climate regime interval and $\Gamma[t]$ is the estimated gamma cdf (Bartoszynsky and Niewiadomska-Bugaj, 1996). A reasonable, further refinement of this statement is to ignore the probability space for very short intervals (5 years or less) that would normally be ignored in practice in retrospective analysis. This is accomplished by using a truncated gamma in Equation (1), $\Gamma[t]=\Gamma[t] /(1-\Gamma[5])$, where $t>5$. The corresponding variation of $P(\rho)$ as a function of $t_{1}$ (abscissa) and $t_{2}$ (ordinate) is shown in Figure 5.

The projections in Figure 5 provide quantitative estimates of probability. Thus, it is generally thought that the AMO switched from cool to warm during the 1994-1995 time frame. If we enter Figure 5 with $t_{1}=10$ years (prior to this writing), we find a rather low probability $(<30 \%)$ that the AMO will switch back to its cool phase in less than $t_{2}=5$ years from 2005. For $t_{2}=10$ and 15 years, the risk increases to $\sim 51$ and $\sim 70 \%$, respectively, and a regime shift within 20 years is highly likely $(\sim 86 \%)$. Such a shift, when it occurs, would imply a return to more frequent droughts in Florida, fewer droughts in the Colorado River basin, and less frequent severe hurricanes in the tropical Atlantic (Enfield et al., 2001; Goldenberg et al., 2001; McCabe et al., 2004). As expected, Figure 5 shows that the risk for any of these $t_{2}$ values increases as time advances and the last regime shift (1994-1995) recedes further into the past ( $t_{1}$ increases).

Somewhat unintuitively, we see that as $t_{1}$ increases, the probability of a future climate shift does not increase very quickly for short-term horizons (small $t_{2}$ ). Thus, for $t_{1}=20$ and 30 years, the probability at $t_{2}=5$ years has only increased to $39 \%$ and $61 \%$, respectively. This is a property of conditional distributions because the probability must asymptote to the $t_{1}$ axis. What changes more noticeably with $t_{1}$, however, is how the probabilities compress into shorter horizon intervals, i.e. the probability increases at a higher rate for longer horizons. An easier way to see this is to plot the horizon $\left(t_{2}\right)$ as a function of $t_{1}$ and the risk (probability) (Figure 6). Thus, for a relatively high-risk level of $70 \%$, the horizon is less than 6 years when $t_{1}=30$ years, as compared to a horizon of 15 years when $t_{1}=10$ years. If $t_{1}=30$, a regime shift is virtually assured within a 10-year horizon.

For any particular application, managers or decision makers may feel comfortable adopting a definition of what constitutes an 'imminent condition' for a regime shift. Thus, for example, one might decide that the point at which a horizon $t_{2}=5$ years achieves a risk level of $70 \%$ constitutes imminence. Referring to Figure 6, we find this to be the case when $t_{1}=30$ years, which will be realized 20 years beyond 2005. For other applications, imminence might be achieved sooner. Clearly, however, there is a finite

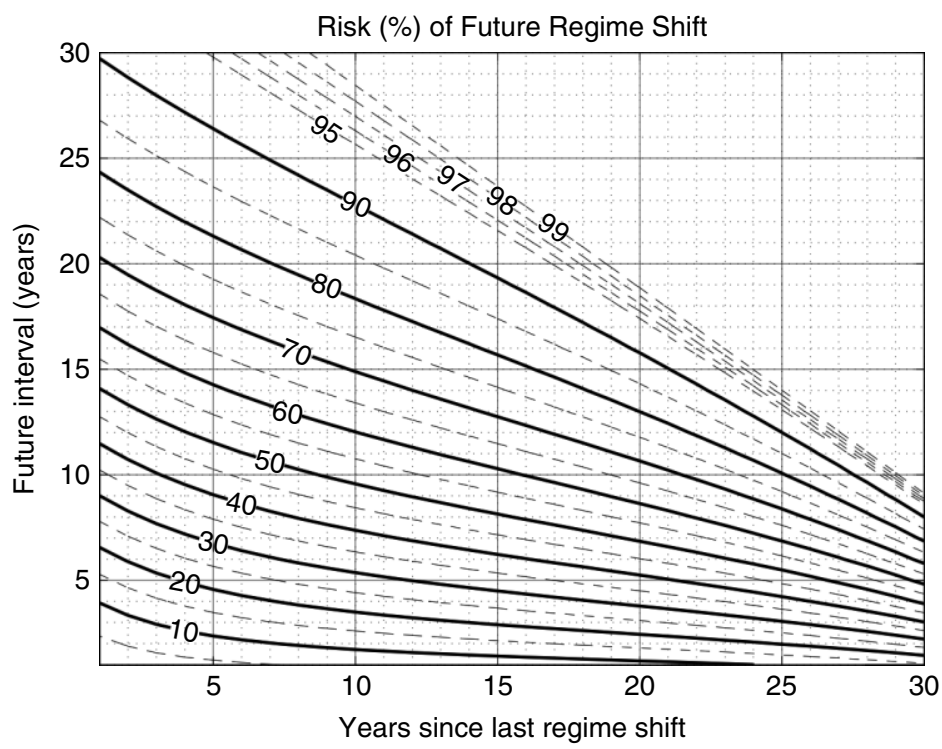

Figure 5. Distribution of the probability of an AMO regime shift occurring within $t_{2}$ future years (ordinate) given that $t_{1}$ years (abscissa) have elapsed since the last regime shift. Based on the gamma distribution with scale and shape parameters of 10.3 years and 1.93 , truncated for $t_{2}+t_{2}>5$ years (see text) 


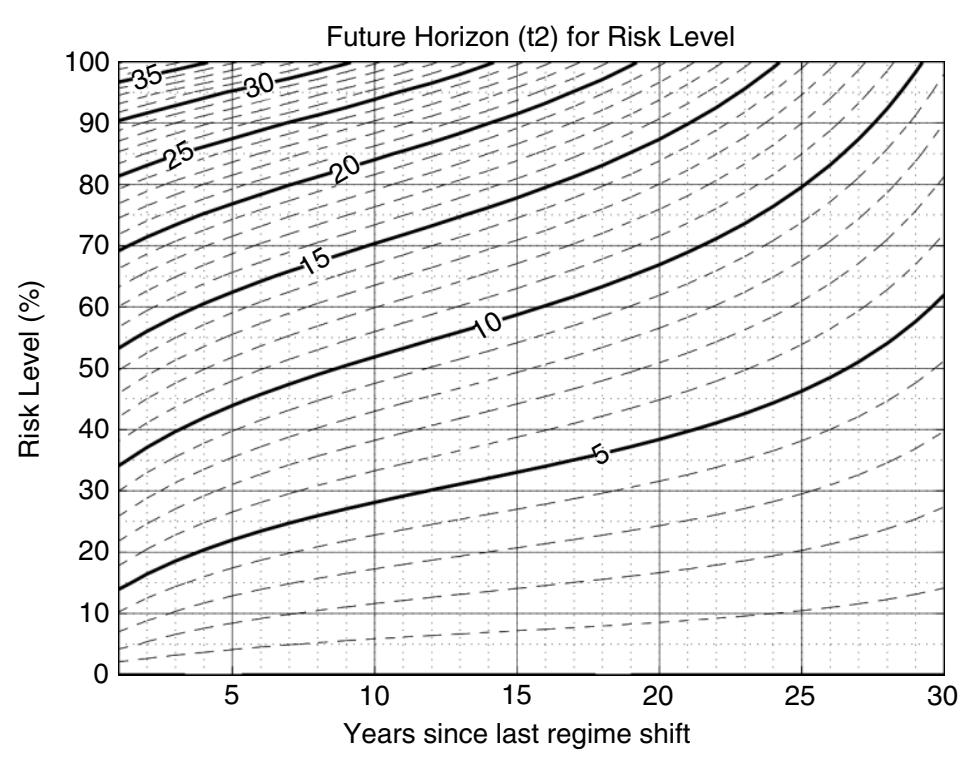

Figure 6. Distribution of the horizon ( $\left.t_{2}\right)$ for an AMO regime shift as a function of risk level (\%, ordinate) given that $t_{1}$ years (abscissa) have elapsed since the last regime shift. Based on the gamma distribution with scale and shape parameters of 10.3 years and 1.93 , truncated for $t_{2}+t_{2}>5$ years (see text)

probability that the regime shift could occur before that point; the definition of imminence should be one for which the immediate implementation of mitigation measures reaches a priority that eclipses most competing considerations. Planning for the contingency of an earlier regime shift should probably be in place considerably before imminence is realized.

\section{UNCERTAINTIES}

The uncertainty of such estimates can be derived from the parameter estimates of the three Gray et al. (2004) time segments, which collectively have a considerably larger spread than those of the 424-year estimation used for Figure 5. This is primarily because of the nonstationarity of the intervals over the last half millennium (Figure 4). Pooling the $3 \times 50$ segment estimates of $A$ and $B$, we then randomly select a large number of parameter values within their overall $1-\alpha$ confidence intervals and generate the corresponding rms uncertainty in $P(\rho)$ over the domain of Figure 5 . The uncertainty is fairly uniform over the $\left[t_{1}, t_{2}\right]$ domain shown. For confidence intervals between $95 \%$ and $99 \%$, the uncertainty ranges between $\pm 2 \%(\alpha=0.05)$ and $\pm 5 \%$ $(\alpha=0.01)$, respectively.

The existence of nonstationarity does not exhaust the sources of uncertainty that attend such projections. For example, it is also desirable to consider how the quality of the reconstruction will affect the distribution parameters. Tree-ring proxies suffer from declining sample-depth backward through time and age-related calibration bias, while different ensembles of trees invariably yield varying results. Where multiple reconstructions of the same climate index are available (at least four exist for the PDO), the uncertainty due to the inability of the reconstructions to perfectly emulate the climate process can be estimated by applying the above methods to the multiple reconstructions, rather than to segments of a single reconstruction. Only one reconstruction yet exists for the AMO, so we have not done this.

Figure 5 is only one example of a potentially useful climate risk projection tool. Thus, for any given year in which decisions are made, one can also construct a graph showing the distribution for $P\left(t_{a}<T \leq t_{b}\right)$, where $t_{a}$ (abscissa) and $t_{b}$ (ordinate) define a time range, e.g. 10-15 years into the future. The risk of an AMO shift between $t_{a}=$ A.D. 2015 and $t_{b}=$ A.D. 2020 is about $19 \%$. This result can also be obtained from Figure 5 by subtraction. 
Other, more complex projections can be developed. McCabe et al. (2004) have shown how the uncorrelated $+/-$ phases of the PDO and AMO have juxtaposed since the mid-nineteenth century in ways that plausibly explain mega droughts in the southwestern and midwestern United States. If both oscillations can be statistically modeled as we have done here only for the AMO, it is possible to develop joint probability projections for the four possible phase-phase scenarios $(+/+.+/-., /-. /+)$, under the assumption that the climate oscillations are mutually independent. It is also possible to query the conditional probability for regime interval magnitude (the area subtended between zero crossings) or intensity (magnitude divided by interval length) given an interval of a certain length. Many of these ideas have already been explored by Biondi et al. (2002, 2005)

In using a proxy index, one must keep in mind that a reconstruction cannot reproduce all of the variance in the instrumental series used for calibration. The smoothed Gray et al. reconstruction accounts for $88 \%$ of the variance in the instrumental AMO index after 1900 when ocean thermometry came into its own. Some of the missing variance affects the interval lengths on which the methods described here are based. Part of the error variance is probably attributable to the insensitivity of tree rings to water surpluses as compared to water deficits; when water is plentiful, growth is limited by other factors such that ring width becomes an underestimate of the true water availability. While this may have only a minimal effect on regime intervals, it can bias any probability method based on magnitude or intensity. The risk methodology, as well as the proxy information per se, must be used, interpreted and applied with care. Thus, the results of McCabe et al. (2004) imply that water managers in the Colorado River Basin should have more confidence in the interpretation of the positive phase of the AMO in terms of drought, than of the negative phase in terms of water surplus.

\section{SUMMARY AND DISCUSSION}

We have shown how a multicentury proxy reconstruction of a climate index may be used to estimate the pdf of climate regime intervals, thus providing a basis for the projection of climate risk and the eventual development of useful decision support tools. The spectrum preserving resampling of the time series provides sufficient sample sizes for pdf estimation using the gamma distribution. Application of the methods to several time segments of the data allow an assessment of stationarity and enable us to estimate the associated uncertainty in the distribution parameters. We find the AMO to have been nonstationary over the last half millennium and the associated uncertainty in probability to be in the range of $2-5 \%$. Finally, we give a detailed example of a derived, climate risk projection and suggest others that can be developed.

Consider the situation in 1990, more than 20 years into a period of cool North Atlantic SSTs (AMO) associated with dry conditions in Florida, wet conditions in the southwestern region and less frequent hurricanes. It is not difficult to imagine management decisions that could have been made then as an AMO reversal became imminent within operational time horizons. Where water was expected to become more plentiful, flood control measures could have been implemented and development on flood plains discouraged. Where more persistent and/or frequent droughts were expected, more water could have been shunted to aquifer storage, water access leases shortened, reservoir withdrawals reduced, conservation measures implemented and agricultural practices modified. These suggestions come from water management and state engineering offices with relevant responsibility for water planning. One might speculate, moreover, that underwriting associations in cooperation with legislators could have increased the funding of windstorm contingency pools in anticipation of more frequent, destructive hurricanes, had such pools existed (they did not). It should be added that in any application, there may be operational or institutional constraints that limit the extent to which risk projection methods may be incorporated into mitigation efforts.

D2M climate risk assessment is not only useful when a climate shift becomes imminent. In general, for any policy or measure that can be adopted in anticipation of a change, there exists an alternative to be followed if the probability of change is low. Policies may be reviewed periodically in light of changing probabilities and the spectrum and effectiveness of available mitigation measures can be revised on a regular basis. Cognizance of the changing nature of climate and its impacts is a relatively recent development and it has taught us that effective management should not be based on static policies. Perhaps the best example 
of this lesson is the recent increase in destructive hurricane potential - related to the change in the AMO climate regime (Goldenberg et al., 2001) - and its impact on the insurance industry.

It is important to point out that the usefulness of these methods for actual applications will depend on the nature of the application, the strength of the connection between the climate mode and the target variable, and managers' ability to utilize the projections in making operational decisions. In general, the closer the relationships of the modeled index to the decision-triggering target variables, the better. Thus, if a proxy reconstruction of stream flow exists, this may be more useful to model than the climate mode whose association with the stream flow is less than perfect. However, projections based on a climate mode have the advantage of being appropriate over a wider range of applications and geographic regions.

Finally, the ultimate uncertainty for which there is no sure remedy at present, is the effect that global climate change will have on future climate regime characteristics. However, it is worth noting that if the true future distribution parameters are different from those in the past, the effect on risk projection (as shown in Figure 5) is to shift all probabilities in the same direction and by similar amounts. Hence, the relative change in probability from one part of the domain to another is little affected by a parameter discrepancy. Arguably, the evolving change in risk is more likely to influence management and policy adjustments, than is the absolute risk at a given position, as long as the errors are within reasonable bounds. In fact, this principle applies to all sources of uncertainty. Thus, the potential importance of climate change need not invalidate the use of paleo-proxy records to project future climate risk, and such indices of past variability should continue to inform management decisions.

\section{ACKNOWLEDGEMENTS}

This research has been supported by the National Oceanic and Atmospheric Administration's (NOAA) Office of Oceanic and Atmospheric Research (OAR) and Office of Global Programs (OGP). The constructive comments of two anonymous reviewers are greatly appreciated. The findings and conclusions in this report are those of the authors and do not necessarily represent the views of the funding agency.

\section{REFERENCES}

Andronova NG, Schlesinger ME. 2000. Causes of global temperature changes during the $19^{\text {th }}$ and $20^{\text {th }}$ centuries. Geophysical Research Letters 27: 2137-2140.

Bartoszynsky R, Niewiadomska-Bugaj M. 1996. Probability and Statistical Inference. Wiley: New York; 826.

Biondi F, Kozubowski TJ, Panorska AK. 2002. Stochastic modeling of regime shifts. Climate Research 23: 23-30.

Biondi F, Kozubowski TJ, Panorska AK. 2005. A new model for quantifying climate episodes. International Journal of Climatology 25: $1253-1264$.

Delworth TL, Mann ME. 2000. Observed and simulated multidecadal variability in the Northern Hemisphere. Climate Dynamics 16: 661-676.

Ebisuzaki W. 1997. A method to estimate the statistical significance of a correlation when the data are serially correlated. Journal of Climate 10: 2147-2153.

Efron B. 1979. Bootstrap methods: Another look at the jackknife. Annals Of Statistics 7: 1-26.

Enfield DB, Mestas-Nuñez AM, Trimble PJ. 2001. The Atlantic multidecadal oscillation and its relation to rainfall and river flows in the continental U.S. Geophysical Research Letters 28: 2077-2080.

Folland CK, Parker DE, Colman AW, Washington R. 1999. Large scale modes of Ocean surface temperature since the late nineteenth century. In Beyond El Niño: Decadal and Inter-decadal Climate Variability, Navarra A (ed). Springer-Verlag: Berlin; 73-102.

Gershunov A, Barnett TP. 1998. Interdecadal modulations of ENSO teleconnections. Bulletin of the American Mathematical Society 79: $2715-2725$.

Goldenberg SB, Landsea CW, Mestas-Nuñez AM, Gray WM. 2001. The recent increase in Atlantic hurricane activity: Causes and implications. Science 293: 474-479.

Gray ST, Graumlich JL, Betancourt JL, Pederson GT. 2004. A tree-ring based reconstruction of the Atlantic Multidecadal oscillation since 1567 A.D. Geophysical Research Letters 31: L12205, DOI:10.1029/2004GL019932.

Gu D, Philander SGH. 1997. Interdecadal climate fluctuations that depend on exchanges between the tropics and extratropics. Science 275: $805-807$.

Hurrell JW. 1995. Decadal trends in the North Atlantic oscillation: Regional temperatures and precipitation. Science 269: 676-679.

Knight JR, Allan RJ, Folland C, Vellinga M, Mann ME. 2005. A signature of persistent natural thermohaline circulation cycles in observed climate. Geophysical Research Letters 32: L20708, DOI:10.1029/2005GL024233.

Latif M, Botset ERM, Esch M, Haak H, Hagemann S, Jungclaus J, Legutke S, Marsland S, Mikolajewicz U. 2004. Reconstructing, monitoring and predicting multidecadal-scale changes in the North Atlantic thermohaline circulation with sea surface temperature. Journal of Climate 17: 1605-1614. 
MacDonald GM, Case RA. 2005. Variations in the Pacific decadal oscillation over the past millenium. Geophysical Research Letters 32: L08703, DOI:10.1029/2005GL022478.

Mantua NJ, Hare SR, Zhang Y, Wallace JM, Francis RC. 1997. A Pacific interdecadal climate oscillation with impacts on salmon production. Bulletin of the American Mathematical Society 78: 1069-1079.

Marshall J, Kushnir Y, Battisti D, Chang P, Czaja A, Dickson R, Hurrell J, McCartney M, Saravanan R, Visbeck M. 2001. North Atlantic climate variability: Phenomena, impacts and mechanisms. International Journal of Climatology 21: 1863-1898.

McCabe GJ, Dettinger MD. 2000. Decadal variations in the strength of ENSO teleconnections with precipitation in the western United States. International Journal of Climatology 19: 1399-1410.

McCabe GJ, Palecki MA, Betancourt JL. 2004. Pacific and Atlantic Ocean influences on multidecadal drought frequency in the United States. Proceedings of the National Academy of Sciences of the United States of America 101: 4136-4141.

Power S, Casey T, Folland C, Colman A, Metha V. 1999. Inter-decadal modulation of the impact of ENSO on Australia. Climate Dynamics 15: 319-324.

Schlesinger ME, Ramankutty N. 1994. An oscillation in the global climate system of period 65-70 years. Nature 367: 723-726.

Sutton RT, Hodson LR. 2005. Atlantic forcing of North American and European summer climate. Science 309: 115-118.

Thompson DWJ, Wallace JM. 1998. The Arctic oscillation signature in the wintertime geopotential height and temperature fields. Geophysical Research Letters 25: 1297-1300.

Woodhouse CA, Gray ST, Meko DM. 2006. Updated streamflow reconstructions for the upper Colorado River Basin. Water Resources Research In press. 